\title{
Calcium Carbonate Mineralization in a Confined Geometry
}

\author{
Gábor Schuszter, Fabian Brau, and A. De Wit*
}

1

Université libre de Bruxelles (ULB), Nonlinear Physical Chemistry Unit, CP231, 1050 Brussels, Belgium

E-mail: adewit@ulb.ac.be

Phone: +32 2650 5774. Fax: +3226505767

\begin{abstract}
Injection of $\mathrm{CO}_{2}$ in porous aquifers, where mineralization takes place via chemical reactions, is one possible long-term solution considered for storage of this greenhouse gas. This mineralization is investigated here experimentally in a confined geometry by injecting radially an aqueous solution of carbonate into a solution of calcium ions to produce solid calcium carbonate. Various precipitation patterns are observed depending on the injection rate and concentrations of the reactants. The pattern properties are quantified to analyze the influence of the growth conditions on the mineralization. We show the existence of critical concentrations of reactants, which are functions of the flow rate, above which the amount of precipitate drops significantly.
\end{abstract}

\section{Introduction}

To reduce atmospheric concentrations of $\mathrm{CO}_{2}$, a possible solution is its sequestration in depleted oil ${ }^{1}$ or gas reservoirs, or in saline aquifers. ${ }^{2}$ In such aquifers, transport and

${ }^{*}$ To whom correspondence should be addressed 
reactive processes take place during the $\mathrm{CO}_{2}$ plume migration, such as wormholing ${ }^{3}$ and precipitation. ${ }^{4,5}$ Mineral carbonation is particularly interesting since it offers a safe long-term storage of $\mathrm{CO}_{2}$. In this mineralization, carbonates resulting from dissolution of

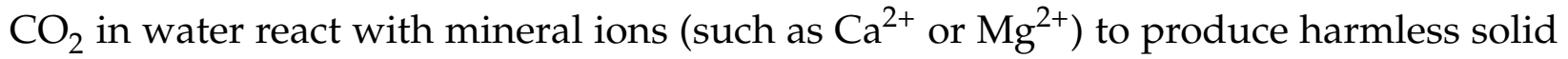
precipitates trapped in the soils.

Such a precipitation at the miscible interface between two reactive solutions can lead to fingering in porous media due to a local decrease in permeability. ${ }^{6} \mathrm{It}$ is of interest to understand how such fingering can affect the efficiency of $\mathrm{CO}_{2}$ mineralisation in terms of the amount of solid phase produced and of its spatial distribution. However, insight into such problematics is difficult to assess in situ.

In this context, we study here experimentally precipitation patterns of calcium carbonate $\left(\mathrm{CaCO}_{3}\right)$ by radial injection in a horizontal confined reactor ${ }^{7-10}$ of a solution of carbonates $\left(\mathrm{CO}_{3}^{2-}\right)$ into a solution of calcium ions $\left(\mathrm{Ca}^{2+}\right)$. This mimics the situation where carbonates resulting from dissolution of $\mathrm{CO}_{2}$ in water react with mineral ions present in that water. The precipitate is produced by the reaction $\mathrm{Ca}_{(\mathrm{aq})}^{2+}+\mathrm{CO}_{3(\mathrm{aq})}^{2-} \rightleftharpoons \mathrm{CaCO}_{3(\mathrm{~s})}$. A large variety of patterns is obtained when the reactant concentrations and flow rate are varied. We characterize quantitatively the area of the pattern covered by the precipitate, its radial extent and its spatial distribution. We find that the amount of precipitate varies with the injection speed and the concentration of reactants, the smallest amount being produced at high values of these parameters.

\section{Materials and methods}

Experiments are performed in a horizontal Hele-Shaw cell consisting of two parallel transparent Plexiglass plates $(19.5 \mathrm{~cm} \times 19.5 \mathrm{~cm})$ separated by a small interstice $(0.5 \mathrm{~mm})$. The cell is illuminated from above by two light pads placed symmetrically with respect to a digital camera recording the dynamics. The cell is initially filled by an aqueous 
solution of $\mathrm{CaCl}_{2}$. An aqueous solution of $\mathrm{Na}_{2} \mathrm{CO}_{3}$ is injected radially from the center of the lower plate through an inlet of $1 \mathrm{~mm}$ inner diameter using a syringe pump. The $\mathrm{pH}$ of this solution is adjusted to 10 by addition of $2 \mathrm{~mol} / \mathrm{L} \mathrm{HCl}$ to avoid the production of solid calcium hydroxide $\mathrm{Ca}(\mathrm{OH})_{2}$. The injection is performed at a constant flow rate $Q$ in the range of those used for displacements in well-bore samples. ${ }^{4}$ The two other control parameters are the normalized concentrations of reactants, $[X]_{n}=c_{x} / c_{\max }$, which are their dimensional concentrations $c_{x}$ divided by their solubility in water, $c_{\max }$ at $T=(21 \pm 1)^{\circ} \mathrm{C}$ (Table S1 in Supporting Information).

Fig.1(a-c) shows a representative time sequence of the precipitation dynamics. At the beginning of radial injection, the contact zone between the two solutions grows as an expanding circle (Fig. 1(a)). The formation of the precipitate decreases locally the mobility of the reactive zone displaced by the injected solution of higher mobility. This unstable situation ${ }^{6}$ leads to the emergence of fingers (Fig. 1(b)). Depending on the contrast in mobility, the amplitude of this destabilization may be small leading to almost circular patterns or large with pronounced fingers and a non uniform spatial distribution.

To study the effect of initial reactant concentrations and flow rate on precipitate pattern formation, a quantitative image analysis is performed. We measure the grayscale value, $I(x, y, t)$, of the images of the dynamics where $x$ and $y$ are spatial coordinates. We compute a normalized intensity $I_{n} \in[0,1]$ as $I_{n}(x, y)=\left[I(x, y)-I_{\text {back }}(x, y)\right] /\left[I_{\max }-I_{\min }\right]$, where $I_{\text {back }}(x, y)$ is the grayscale intensity of a background image (taken before the experiment starts) and $I_{\max }$ and $I_{\min }$ its maximum and minimum values. Finally, $I_{n}(x, y)$ is set to 0 if it is smaller than some threshold $I_{\text {th }}=0.05$. This threshold fixes the smallest intensity below which we cannot determine with certainty the presence of a precipitate. An example of the spatial distribution of $I_{n}(x, y, t)$ is shown in Fig.1(d). The radius $R_{\max }$ is the largest radial distance between the injection point and the pattern perimeter. For each image, we also compute: (i) the area $A$ covered by the pattern (Fig.1(e)) i.e. the area where $I_{n}>I_{\text {th }}$; (ii) the area $A_{\mathrm{p}}$ of the zone inside the pattern perimeter (Fig.1(f)). 
We next define four quantities to measure the pattern properties. The first quantity is the total grayscale intensity, $I_{\text {tot }}(t)=\int I_{n}(x, y, t) d x d y \in[0, A]$ where $0<A<N=1280 \times$ 1024 pixels of the image resolution. Even though a given $I_{\text {tot }}$ might correspond to various precipitate thicknesses within the cell, it qualitatively indicates the amount of precipitate in the gap thanks to its white color. The second quantity is the brightness of the precipitate, $B=I_{\text {tot }} / A$, normalizing its total grayscale intensity $I_{\text {tot }}$ by the area $A$ it is covering (Fig.1(e)). $B$ varies between 0 and 1 with values close to 1 indicating a concentrated bright precipitate. The third quantity is the filling of the pattern, $F=A / A_{\mathrm{p}} \in[0,1]$, dividing its area $A$ by the area $A_{\mathrm{p}}$ enclosed by its perimeter (Fig.1(f)). $F$ is smaller than 1 if the pattern contains some black areas without precipitate. A case for which $F \ll 1$ indicates a hollow mineralization structure. The last quantity is the pattern density, $d=A_{\mathrm{p}} /\left(\pi R_{\max }^{2}\right) \in[0,1]$, comparing the area $A_{\mathrm{p}}$ of the pattern to the area of the circle of radius $R_{\max }$ (Fig.1(d)). ${ }^{10} \mathrm{It}$ measures deviations from a symmetric radial growth (for which $d=1$ ). Low values of $d$ indicate that precipitation occurs in some preferred directions.

\section{Results and discussion}

Figure 2 shows the various patterns obtained under different experimental conditions after injecting $3 \mathrm{~mL}$ of $\mathrm{CO}_{3}^{2-}$ solution into the $\mathrm{Ca}^{2+}$ solution. Considering the concentrations (Table S1 in Supporting Information) and the activity coefficients for the related solutions, ${ }^{11,12}$ the saturation index $\mathrm{SI}=\log _{10}\left(\mathrm{IAP} / K_{S P}\right)$ can be calculated as being in the range $6.8-9.1$ (Table S2 in Supporting Information). Here IAP and $K_{S P}$ are the ion activity product of the reactant mixture and the solubility product of $\mathrm{CaCO}_{3}$, respectively. The high SI values show that the reactant mixture is highly supersaturated in the localized reaction zone at the interface between the carbonate and calcium solutions. Precipitation occurs thus fast and the Damköhler number (ratio of hydrodynamic to chemical time scales) is expected to be large in each case. 
Let us first examine the patterns obtained for various flow rates at the lowest concentrations for both reactants. At the lowest flow rate (Fig.2(a7)), a circular structure grows homogeneously. The amount of precipitate is small which gives a low brightness $B=0.13$. The $F=1$ value indicates that some precipitate is produced everywhere within the pattern perimeter as for all patterns at the lowest flow rate. Small radial stripes appear at the periphery when $Q$ is increased (Fig.2(b7), Movie S1). They originate from buoyancy-driven convection due to density gradient. ${ }^{13}$ The radial symmetry is however preserved but the precipitate starts to be flushed away of the injection point by the flow; $F$ is thus slightly smaller than 1. Increasing further the flow rate destabilizes the pattern perimeter in fingers which break the radial symmetry (Fig.2(c7)). The enhanced flow advects the precipitate to the periphery of the structure leading to a smaller value of $F$. In some cases, spirals can be observed (Fig.2(c5), Movie S2) similar to those studied for other chemicals. ${ }^{8}$

Keeping the concentration of one of the reactant at its lowest value and increasing the concentration of the other reactant leads to a larger amount of precipitate and hence to an increase of $I_{\text {tot }}$ and $B$. The effect on $I_{\text {tot }}$ is more pronounced when the concentration of carbonate is increased at fixed calcium concentration than in the reverse case (Fig.3). This could be explained by the relatively larger viscosity change of $\mathrm{Ca}^{2+}$ solution (see Table S1) as the concentration increases which hinders the mixing of the reactants when the less viscous $\mathrm{CO}_{3}^{2-}$ is injected. Influence of various mixing effects due to the flow ${ }^{7,14}$ on underlying reaction-diffusion profiles ${ }^{6}$ should also be investigated to better understand these differences.

The evolution of $I_{\text {tot }}$ as a function of the flow rate is shown in Fig.4(a,b). One might intuitively expect that the regime where most precipitate is produced is reached when the concentrations of both reactants are large. This is however not the case. At a large enough constant flow rate $Q$, increasing the concentration of both reactants leads to patterns showing barely any precipitate (Fig.2(b3) and (c2,3)) even though the SI is high in the reaction zone. These patterns take the form of wide hollow tubes because the 
injected carbonate solution channels through the calcium one without much mixing and precipitation. Similarly, at given large enough concentrations of both reactants, increasing the flow rate $Q$ leads to a strong decrease of the $B$ value and $I_{\text {tot }}$ (Fig.4(c)) indicating a sharp drop in the mineralization.

To understand the evolution of these hollow patterns, note that the ratio between the viscosity of the displaced $\mathrm{Ca}^{2+}$ and that of the injected $\mathrm{CO}_{3}^{2-}$ solution is only about 3 (Table S1). As shown previously ${ }^{10}$, this viscosity contrast is not large enough to produce viscous fingering in our experimental conditions. However, as soon as the reactants are in contact, precipitation occurs along the flow producing a cohesive precipitate barrier between the two reactants with a random shape (Fig.2(b3) and (c2,3)). New precipitate layers next form along the fast moving liquid-liquid interface giving precipitate walls which thicken and get more rigid (Movie S3). The stiffness of these walls allows to sustain the interior pressure inhibiting further mixing of the reactant solutions. Later on the barrier thickens then only slowly from secondary reaction-diffusion growth on a much longer timescale (hours compared to $28 \mathrm{~s}$ of experimental time in the case of $Q=6.5 \mathrm{~mL} / \mathrm{min}$ ). These tube-like patterns are characterized by low values of $d$ due to an asymmetric geometry.

The various trends observed in Fig. 2 can be summarized as follow: (i) Homogeneous circular patterns are obtained at low concentrations and low flow rates $Q$. In that case, the brightness $B$ is small and the filling $F$ is close to 1 which indicates that there is a small amount of precipitate everywhere inside the perimeter with a pattern density $d$ close to 1 . When the flow rate $Q$ increases, some fingering appears and $F$ decreases. (ii) If the concentration of one of the two reactants is maintained low and the other one is increased, more precipitate is formed with an increase in $B$. Heterogeneities in the precipitate distribution appear at large $Q$. (iii) For large concentrations of both reactants and beyond some $Q$, we observe hollow tubes with a sharp drop in the production of precipitate even if the SI remains high, and asymmetries in the distribution leading to low 
values of $B, F$ and $d$.

The implications for $\mathrm{CO}_{2}$ storage are the following. For low concentrations of minerals (i.e. $\mathrm{Ca}^{2+}$ here) in the soil, the amount of precipitate increases with the concentration of carbonate (related to the amount of $\mathrm{CO}_{2}$ injected) as shown in Fig.3a, with a slight influence of $Q$ (Fig.4a). For larger concentrations of minerals, the amount of precipitate first increases with the concentration of carbonate before sharply dropping down beyond some critical value $\left[\mathrm{Ca}^{2+}\right]_{n}^{\mathrm{c}}$, which decreases as $Q$ increases (see Fig.2 and 4). At the lowest flow rate $(Q=0.1 \mathrm{~mL} / \mathrm{min})$, this critical concentration $\left[\mathrm{Ca}^{2+}\right]_{n}^{\mathrm{c}}$ has not been reached here. At $Q=1 \mathrm{~mL} / \mathrm{min}$, we find that $0.23<\left[\mathrm{Ca}^{2+}\right]_{n}^{\mathrm{c}}<0.68$ and at $Q=6.25 \mathrm{~mL} / \mathrm{min}, 0.08<$ $\left[\mathrm{Ca}^{2+}\right]_{n}^{c}<0.23$.

Pattern formation during $\mathrm{CaCO}_{3}$ mineralization has been studied experimentally during radial injection of an aqueous solution of carbonate into a solution of $\mathrm{Ca}^{2+}$ within a confined geometry. The amount of precipitate, as well as its spatial distribution, depend on the concentrations of the reactants and on $Q$. The patterns have been characterized by their grayscale intensity, spatial area and perimeter to quantify the amount of minerals produced and the geometry of the pattern. We find that, above critical values of concentrations which depend on the flow rate $Q$, the amount of precipitate drops significantly even if the SI in the localized reaction zone is large. This suggests that the highest efficiency of $\mathrm{CaCO}_{3}$ mineralization is not obtained necessarily at high concentrations and high $Q$. Our analysis paves the way to numerous possible experimental and theoretical extensions. From a theoretical point of view, the effect of flow and mixing on precipitation should be further analyzed to understand in which conditions precipitation drops even if the SI remains large, as observed here. The robustness of our conclusions could be tested experimentally with changes in the reactants, viscosities, pressure, temperature and $\mathrm{pH}$, both in simple Hele-Shaw cells and more complex geometries including Hele-Shaw cells with obstacles or beads to vary the porosity and real 3D porous systems. 


\section{Acknowledgement}

We thank Prodex for financial support.

\section{Supporting Information Available}

Description of solution properties (table S1); saturation index for reactant concentration pairs (Table S2); temporal evolution of the pattern 7 shown in Fig.2(b) (accelerated 10 times, movie S1); pattern 5 shown in Fig.2(c) (accelerated 4 times, movie S2); pattern 3 shown in Fig.2(c) (accelerated 15 times, movie S3).

This material is available free of charge via the Internet at http://pubs . acs . org/.

The authors declare no competing financial interest.

\section{References}

(1) Dai, Z.; Middleton, R.; Viswanathan, H.; Fessenden-Rahn, J.; Bauman, J.; Pawar, R.; Lee, S.-Y.; McPherson, B. An Integrated Framework for Optimizing $\mathrm{CO}_{2}$ Sequestration and Enhanced Oil Recovery. Environ. Sci. Technol. Lett. 2014, 1, 49-54.

(2) Metz, B.; Davidson, O.; de Conninck, H. C.; Loos, M.; Meyer, L. A., Eds. IPCC Special Report on Carbon Dioxide Capture and Storage; Cambridge University Press: New-York, 2005.

(3) Chadam, D.; Hoff, D.; Merino, E.; Ortoleva, P.; Sen, A. Reactive Infiltration Instabilities, J. Appl. Math. 1986, 36, 207-221.

(4) Luquot, L.; Gouze, P. Experimental Determination of Porosity and Permeability Changes Induced by Injection of $\mathrm{CO}_{2}$ into Carbonate Rocks, Chem. Geo. 2009, 265, 148-159.

(5) White, A.R.; Ward, T. $\mathrm{CO}_{2}$ Sequestration in a Radial Hele-Shaw Cell via an Interfacial Chemical Reaction, Chaos 2012, 22, 037114. 
(6) Nagatsu, Y.; Ishii, Y.; Tada, Y.; De Wit, A. Hydrodynamic Fingering Instability Induced by a Precipitation Reaction, Phys. Rev. Lett. 2014, 113, 024502.

(7) Tartakovsky, A. M.; Redden, G.; Lichtner, P. C.; Scheibe, T. D.; Meakin, P. MixingInduced Precipitation: Experimental Study and Multiscale Numerical Analysis, Water Res. Res. 2008, 44, W06S04.

(8) Haudin, F.; Cartwright, J. H. E.; Brau, F.; De Wit, A. Spiral Precipitation Patterns in Confined Chemical Gardens, Proc. Nat. Acad. Sci. (USA) 2014, 111, 17363-17367.

(9) Zhang, C.; Dehoff, K.; Hess, N.; Oostrom, M.; Wietsma, T. W.; Valocchi, A. J.; Fouke, B. W.; Werth, J. C. Pore-Scale Study of Transverse Mixing Induced $\mathrm{CaCO}_{3}$ Precipitation and Permeability Reduction in a Model Subsurface Sedimentary System, Environ. Sci. Technol. 2010, 44, 7833-7838.

(10) Haudin, F.; De Wit, A. Patterns Due to an Interplay Between Viscous and PrecipitationDriven Fingering, Phys. Fluids 2015, 27, 113101.

(11) Staples, B. R.; Nuttall, R. L. The Activity and Osmotic Coefficients of Aqueous Calcium Chloride at 298.15 K, J. Phys. Chem. Ref. Data 1977, 6 385-407.

(12) Robinson, R. A.; Macaskill, J. B. Osmotic Coefficients of Aqueous Sodium Carbonate Solutions at $25^{\circ} \mathrm{C}$, J. Solution Chem. 1979, 8, 35-40.

(13) Haudin, F.; Riolfo, L. A.; Knaepen, B.; Homsy, G. M.; De Wit, A. Experimental Study of a Buoyancy-Driven Instability of a Miscible Horizontal Displacement in a Hele-Shaw Cell, Phys. Fluids 2014, 26, 044102.

(14) De Simoni, M.; Carrera, J.; Sánchez-Vila, X.; Guadagnini, A. A Procedure for the Solution of Multicomponent Reactive Transport Problems, Water Res. Res. 2005, 41, W11410. 

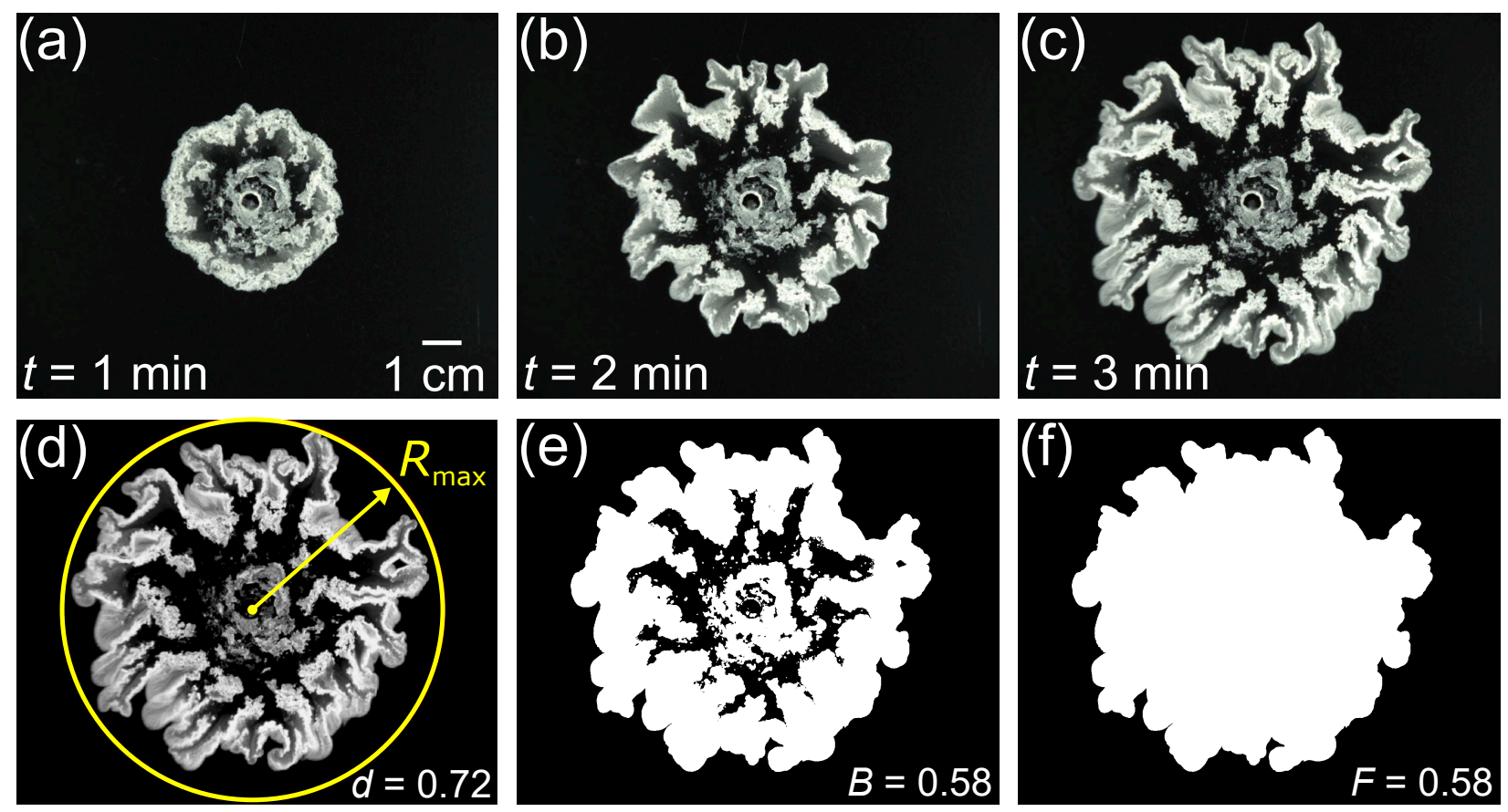

Figure 1: (a)-(c): Time evolution of a fingered precipitation pattern. (d)-(f): Variables used to characterize the patterns. (d) Distribution of grayscale intensity $I(x, y)$. The yellow circle passing by the tip of the longest finger defines the radius $R_{\max }$. (e) Area $A$ covered by the solid phase. (f) Area $A_{\mathrm{p}}$ inside the pattern perimeter.

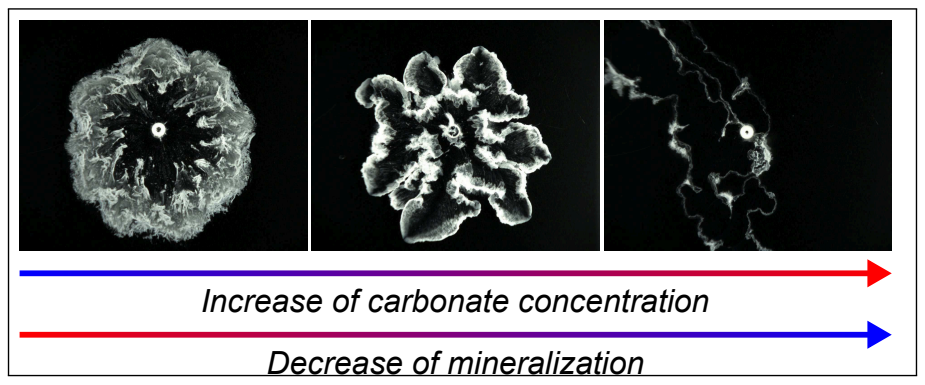



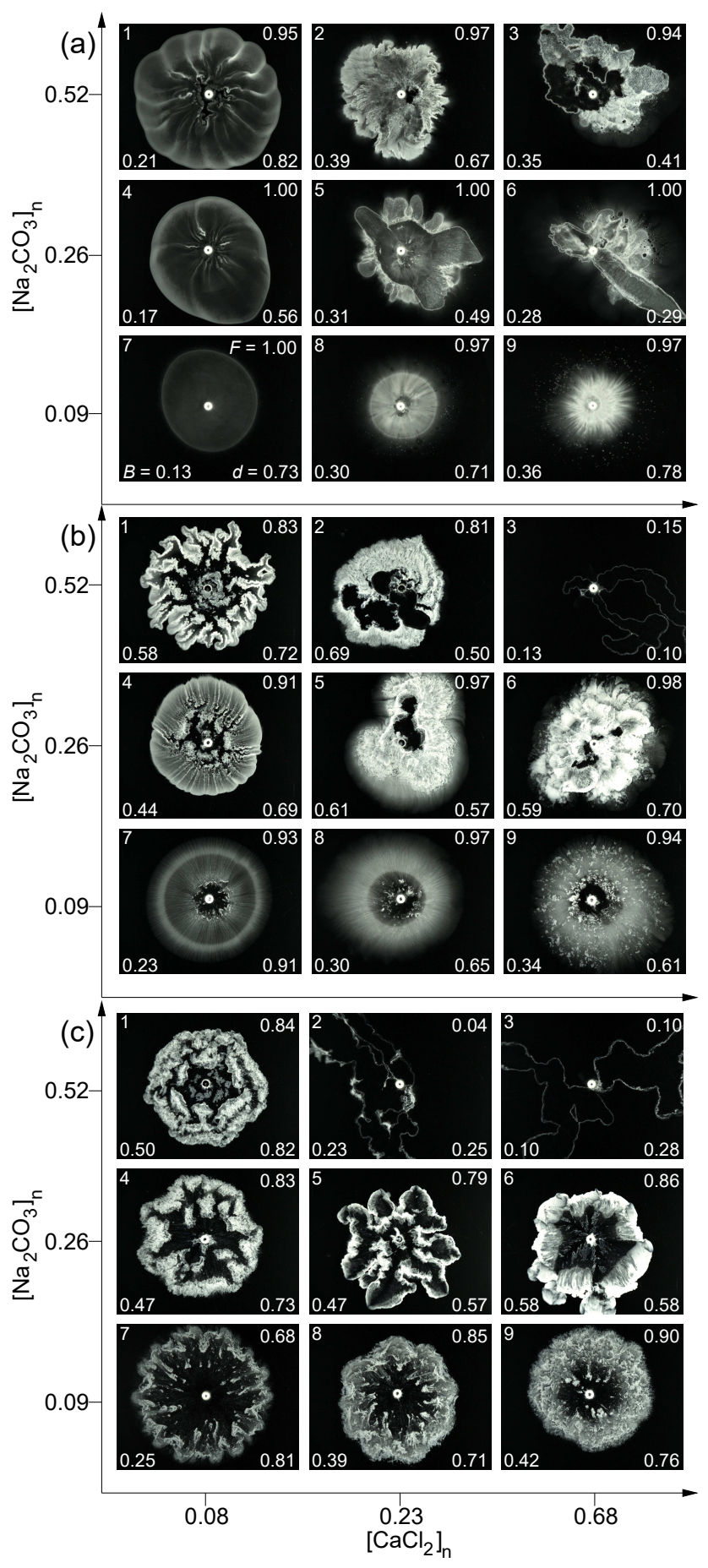

Figure 2: Patterns observed at different flow rates and concentrations. $122 \mathrm{~mm} \times 98 \mathrm{~mm}$ field of view. The numbers in the left top, left bottom, right top, and right bottom corners are, respectively, the pattern number, $B, F$, and $d$ (see pattern a7). (a) $Q=0.1 \mathrm{~mL} / \mathrm{min}$ $(t=30 \mathrm{~min}),(\mathrm{b}) Q=1.0 \mathrm{~mL} / \mathrm{min}(t=3 \mathrm{~min})$, and (c) $Q=6.5 \mathrm{~mL} / \mathrm{min}(t=28 \mathrm{~s})$. 
(a)

(b)
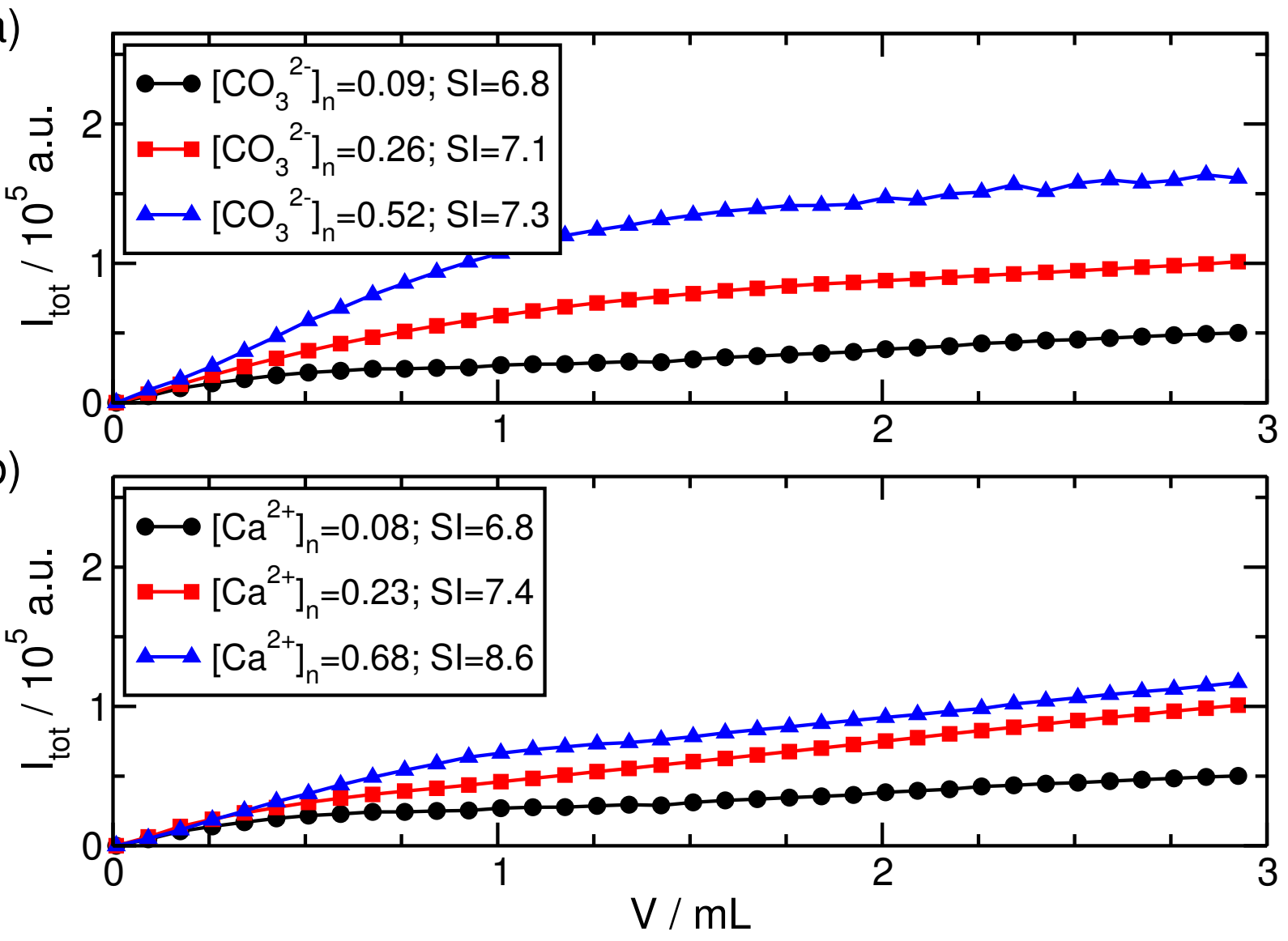

Figure 3: Evolution of $I_{\text {tot }}$ as a function of injected volume $V$ of carbonate solution at $Q=0.1 \mathrm{~mL} / \mathrm{min}$. (a) $\left[\mathrm{Ca}^{2+}\right]_{\mathrm{n}}=0.08$ and $\left[\mathrm{CO}_{3}^{2-}\right]_{\mathrm{n}}$ varies. (b) $\left[\mathrm{CO}_{3}^{2-}\right]_{\mathrm{n}}=0.09$ and $\left[\mathrm{Ca}^{2+}\right]_{\mathrm{n}}$ varies. 


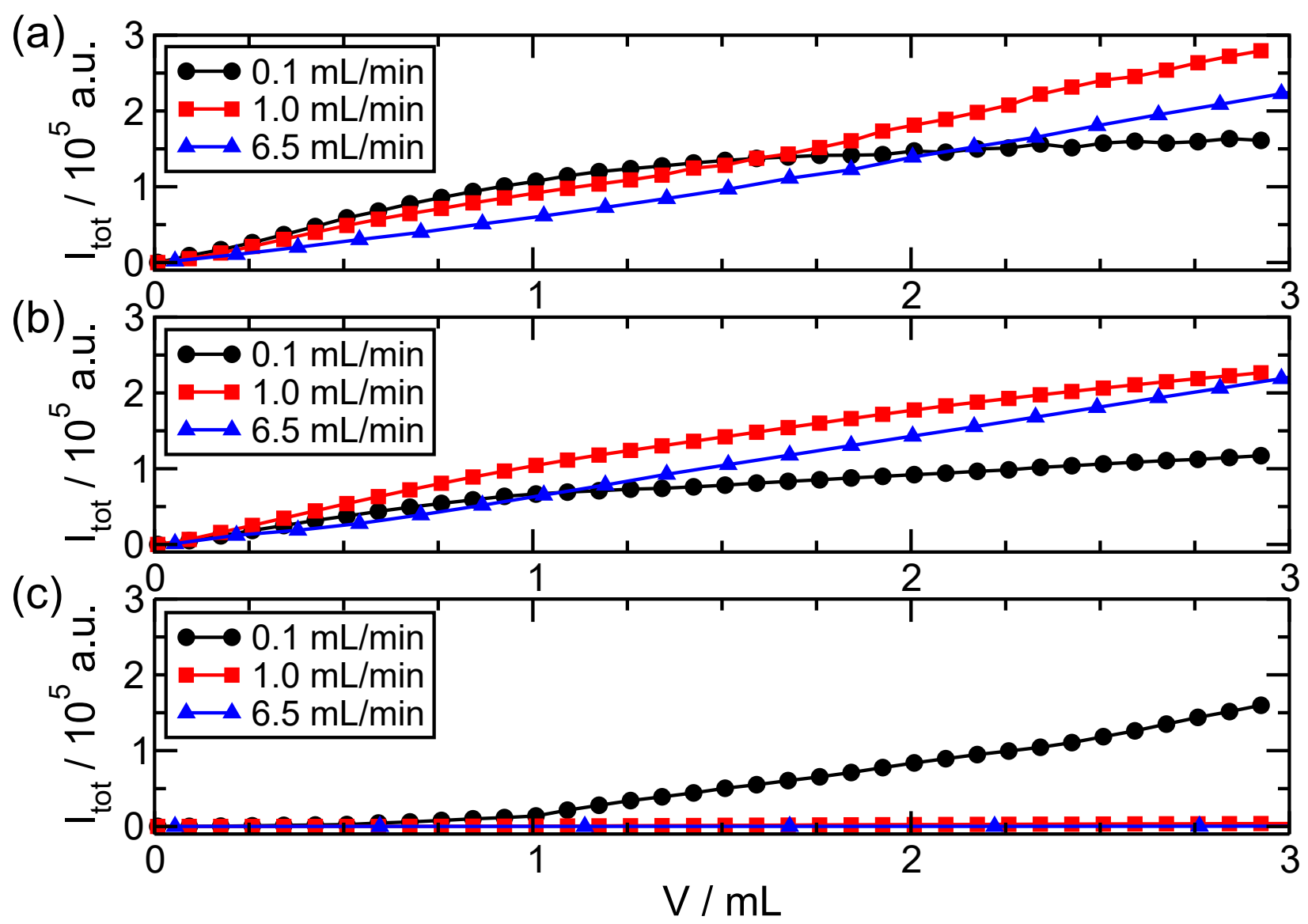

Figure 4: Evolution of $I_{\text {tot }}$ as a function of injected volume $V$ for different flow rates. (a) $\left[\mathrm{Ca}^{2+}\right]_{\mathrm{n}}=0.08,\left[\mathrm{CO}_{3}^{2-}\right]_{\mathrm{n}}=0.52$, and $\mathrm{SI}=7.3$. (b) $\left[\mathrm{Ca}^{2+}\right]_{\mathrm{n}}=0.68,\left[\mathrm{CO}_{3}^{2-}\right]_{\mathrm{n}}=0.09$, and $\mathrm{SI}=8.6 .(\mathrm{c})\left[\mathrm{Ca}^{2+}\right]_{\mathrm{n}}=0.68,\left[\mathrm{CO}_{3}^{2-}\right]_{\mathrm{n}}=0.52$, and $\mathrm{SI}=9.1$. 